\title{
ON DIFFERENCES OF UNITARILY EQUIVALENT SELF-ADJOINT OPERATORS†
}

\author{
by C. R. PUTNAM
}

(Received 20 April, 1959)

1. All operators considered in this paper are bounded operators on a Hilbert space. In case $A$ and $B$ are self-adjoint, certain conditions on $A, B$ and their difference

$$
H=A-B \text {, }
$$

assuring the unitary equivalence of $A$ and $B$,

$$
B=U^{*} A U,
$$

have recently been obtained by Rosenblum [6] and Kato [2]. The present paper will consider the problem of investigating consequences of an assumed relation of type (2) for some unitary $U$ together with an additional hypothesis that the difference $H$ of (1) be non-negative, so that

$$
H=A-B \geqslant 0 \text {. }
$$

First, it is easy to see that if only (2) and (3) are assumed, thereby allowing $H=0$, relation (2) can hold for $A$ arbitrary with $U=I$ (identity) and $B=A$. If $H=0$ in (3) is not allowed, however (an impossible assumption in the finite dimensional case, incidentally, since then the trace of $H$ is zero and hence $H=0$ ), it will be shown, among other things, that any unitary operator $U$ for which (2) and (3) hold must have a spectrum with a positive measure (as a consequence of (i) of Theorem 2 below). Moreover $A$ (hence $B$ ) cannot differ from a completely continuous operator by a constant multiple of the identity (Theorem 1). In case 0 is not in the point spectrum of $H$, then $U$ is even absolutely continuous (see (iv) of Theorem 2). In $\$ 4$, applications to semi-normal operators will be given.

Let $U$ be any unitary operator with the spectral resolution

$$
U=\int e^{i \lambda} d E(\lambda) \quad\left(\int=\int_{0}^{2 \pi}\right)
$$

Let $\left\{e^{i \lambda_{n}}\right\}, 0 \leqslant \lambda_{n}<2 \pi$, denote the point spectrum (if any) of $U$ and put

Then the $E_{c}(\lambda)$ are projections and one can write

$$
E_{\mathrm{c}}(\lambda)=E(\lambda)-\sum_{\lambda_{n}<\lambda}\left\{E\left(\lambda_{n}+0\right)-E\left(\lambda_{n}-0\right)\right\}
$$

$$
U=\sum_{n} e^{i \lambda n}\left[E\left(\lambda_{n}+0\right)-E\left(\lambda_{n}-0\right)\right]+\int e^{i \lambda} d E_{c}(\lambda),
$$

where the integral (if present) represents the continuous component of $U$. In case this component is present and if $\left(E_{c}(\lambda) x, y\right)$ is absolutely continuous for all $x, y$, that is, if $\int_{Z} d E_{c}(\lambda)=0$ for every zero set $Z$, then this component will be called absolutely continuous. The operator

† This research was supported by the United States Air Force through the Air Force Office of Scientific Research and Development Command, under Contract No. AF 18 (603)-139. Reproduction in whole or in part is permitted for any purpose of the United States Goverment. 
$U$ itself will be called absolutely continuous if it has no point spectrum and if its continuous component is absolutely continuous.

Since $A-U^{*} A U$ can be expressed as $U\left(U^{*} A\right)-\left(U^{*} A\right) U$, the commutator of $U$ and $U^{*} A$, relations (2) and (3), that is,

imply, as was shown in [3], that

$$
0 \leqslant H=A-U^{*} A U
$$

$$
H \int_{Z} E(\lambda)=0
$$

where $Z$ denotes an arbitrary zero set.

2. Relation (6) will be used to prove

Theorem 1. Suppose that the self-adjoint operators $A$ and $B$ satisfy (2) and (3) and let $\delta=\delta(A)$ denote the difference of the maximum and minimum points of the essential spectrum of $A$. Then

$$
\|H\| \leqslant \delta
$$

in particular, if $A$ differs from a completely continuous operator by a constant multiple of the identity, then $H=0$.

Here, $\|C\|$ is defined by $\|C\|=\sup \|C x\|$, where $\|x\|=1$, and the essential spectrum of $C$ is the set of cluster points, including points of the point spectrum of infinite multiplicity, of the spectrum of $C$. Incidentally, since, as was remarked above, $H \geqslant 0$ can hold for finite matrices only if $H=0$, it can always be supposed that the basic Hilbert space is infinite dimensional, in which case any self-adjoint operator necessarily has a non-empty essential spectrum.

Proof of Theorem 1. Let $\lambda_{0}$ denote the maximum point in the essential spectrum of $A$ and denote the eigenvalues of $A$ (if any) greater than $\lambda_{0}$ by $\lambda_{1}>\lambda_{2}>\ldots$ If $x_{1}$ is any eigenfunction of $U^{*} A U$ belonging to $\lambda_{1}$ then, by (5),

$$
0 \leqslant\left(H x_{1}, x_{1}\right)=\left(A x_{1}, x_{1}\right)-\lambda_{1}\left(x_{1}, x_{1}\right) \leqslant 0
$$

and so $\left(A x_{1}, x_{1}\right)=\lambda_{1}\left(x_{1}, x_{1}\right)$. Hence $0=\left(\lambda_{1} I-A\right)^{\frac{1}{2}} x_{1}=\left(\lambda_{1} I-A\right) x_{1}$ and so $x_{1}$ is an eigenfunction of $A$ belonging to $\lambda_{1}$. Since $\lambda_{1}$ belongs to the spectra of $A$ and $U^{*} A U$ with the same (finite) multiplicity, it follows that the eigenfunctions of $A$ and $U^{*} A U$ belonging to $\lambda_{1}$ are identical. On treating successively $\lambda_{2}, \lambda_{3}, \ldots$ in a similar manner, it follows that the eigenfunctions of $A$ and $U^{*} A U$ for each of the numbers $\lambda_{n}$ are identical.

Let $\mu_{1}<\mu_{2}<\ldots$ denote the eigenvalues of $A$ (if any) less than the least point $\mu_{0}$ of the essential spectrum of $A$. If $y_{1}$ is an eigenfunction of $A$ belonging to $\mu_{1}$, then one has

$$
0 \leqslant\left(H y_{1}, y_{1}\right)=\mu_{1}\left(y_{1}, y_{1}\right)-\left(U^{*} A U y_{1}, y_{1}\right) \leqslant 0 ;
$$

hence $\left(U^{*} A U y_{1}, y_{1}\right)=\mu_{1}\left(y_{1}, y_{1}\right)$, and so $y_{1}$ must be an eigenfunction of $U^{*} A U$ belonging to $\mu_{1}$. As before, it follows that the eigenfunctions of $A$ and $U A^{*} U$ belonging to eigenvalues $\mu_{n}$ less than $\mu_{0}$ are identical.

It is now easy to complete the proof of the theorem. For if $x$ is any element of Hilbert space, it can be written as $x=z+w$, where $z$ is the projection of $x$ on the space spanned by the eigenfunctions of $A$ belonging to eigenvalues outside the interval $\mu_{0} \leqslant \lambda \leqslant \lambda_{0}$ and $w$ is in the orthogonal complement. Clearly $\mathrm{Hz}=0$ and hence

$$
(H x, x)=(H w, w)=(A w, w)-\left(U^{*} A U w, w\right) \leqslant\left(\lambda_{0}-\mu_{0}\right)\|w\|^{2} \leqslant\left(\lambda_{0}-\mu_{0}\right)\|x\|^{2} .
$$

Relation ( 7 ) follows and the proof of Theorem 1 is complete. 
3. Theorem 2. Suppose that the self-adjoint operators $A$ and $B$ satisfy (2) and (3) and let $N$ denote the multiplicity of the eigenvalue 0 of $H(0 \leqslant N \leqslant \infty)$. Then : (i) If $H \neq 0$, and if $U$ has the spectral resolution (4), then $\int_{Z} d E(\lambda)<I$ for every zero set $Z$. (ii) The point spectrum of $U$ has no more than $N$ values (counting multiplicities). (iii) If $N<\infty$, then the continuous component of $U$ is absolutely continuous. (iv) If $N=0$, then $U$ is absolutely continuous. (v) If $N=0$, the maximum and minimum points of the spectrum of $A$ cannot belong to the point spectrum of $A$ (and hence must belong to the essential spectrum of $A$ ).

Proof of Theorem 2. Assertion (i) is an immediate consequence of (6); cf. [3]. Let $x$ be an eigenfunction of $U$; then, by (5), one has

$$
0 \leqslant(H x, x)=(A x, x)-(A x, x)=0 ;
$$

hence $0=H i x=H x$. This proves (ii). In order to prove (iii) note that, by (ii), $U$ has at most a finite number of points in its point spectrum and so its continuous component is present. But if this component were not absolutely continuous, there would exist a zero set $Z$ and an element $x$ such that $\int d E_{c}(\lambda) x \neq 0$. Clearly $Z$ can be written as $Z=\Sigma Z_{n}$ where $Z_{1}, Z_{2}, \ldots$ denotes an infinite sequence of non-overlapping zero sets for which $x_{n}=\int_{Z_{n}} d E_{\mathrm{c}}(\lambda) x \neq 0$. Thus the $x_{n}$ are orthogonal and, by (6), each is an eigenfunction of $H$ belonging to 0 . Thus $N=\infty$, a contradiction, and (iii) is proved. Assertion (iv) is a consequence of (ii) and (iii). Assertion (v) follows from (5). For if the maximum point $\lambda_{M}$ of the spectrum of $A$ were in the point spectrum of $A$, hence of $U^{*} A U$, then for a corresponding eigenfunction $x$ of $U^{*} A U$ one would have

$$
0<(H x, x)=(A x, x)-\lambda_{M}(x, x) \leqslant 0,
$$

a contradiction. Similarly the minimum point $\lambda_{m}$ cannot be in the point spectrum and the proof of $(v)$ is complete.

It can be remarked that if 0 is not in the point spectrum of $H$, then the proof of Theorem 1 is an immediate consequence of $(v)$ of Theorem 2. For obviously

$$
(H x, x)=(A x, x)-\left(U^{*} A U x, x\right) \leqslant\left(\lambda_{M}-\lambda_{m}\right)\|x\|^{2} .
$$

4. Applications to semi-normal operators. Let $D$ be an arbitrary (bounded) operator and consider

$$
H=D D^{*}-D^{*} D \text {. }
$$

If $H$ is semi-definite (in which case, only $H \geqslant 0$ will be supposed), $D$ is called semi-normal. In case $D$ is non-singular, it has a polar decomposition $D=P U$ where $P$ is positive selfadjoint and $U$ is unitary. Then $D D^{*}=P^{2}, D^{*} D=U^{*} P^{2} U$ and (8) can be written as $H=P^{2}-U^{*} P^{2} U$, so that $P^{2}$ can be identified with the $A$ considered above. Of course, it is quite possible that $D^{*} D=U^{*}\left(D D^{*}\right) U$ holds for some unitary $U$ even if $D$ is singular.

It was shown in [4] that the spectra of the real and imaginary parts of a semi-normal, but not normal, operator $D$ (in fact, the spectra of $\frac{1}{2}\left(e^{-i \theta} D+e^{i \theta} D^{*}\right)$ for $\theta$ arbitrary and real) are of positive measure. In case $D$ is non-singular with the polar decomposition $D=P U$ then, as a consequence of (i) of Theorem 2, it follows that $U$ also has a spectrum of positive measure. However, a similar claim cannot be made for the positive operator $P$. In fact, as is shown by 
Theorem 3 below and the example following, $P$ must have at least two points in its essential spectrum, and may possibly have only (these) two points in its spectrum.

As a corollary of Theorem 1, one has

Tнеовем 3. If $H$ defined by (8) satisfies $H \geqslant 0$, and if $D D^{*}$ and $D^{*} D$ are unitarily equivalent, then (7) holds, where $\delta=\delta\left(D D^{*}\right)$ is the difference of the maximum and minimum points of the essential spectrum of $D D^{*}$. Thus, if in addition, $H \neq 0$, then $\delta\left(D D^{*}\right)>0$ and $D D^{*}$ (hence $D^{*} D$ ) cannot differ from a completely continuous operator by a multiple of the identity.

It is easy to show that the inequality (7) occurring in Theorems 1 and 3 may become an equality and that $A$ may have only two points in its spectrum. One need only choose $A=\left(a_{i j}\right)$ and $B=\left(b_{i j}\right)$, where $i, j=0, \pm 1, \pm 2, \ldots$, to be doubly infinite matrices for which $a_{i i}=1$ if $i=0,1,2, \ldots$ and $a_{i j}=0$ otherwise, and $b_{i i}=1$ if $i=1,2, \ldots$ and $b_{i j}=0$ otherwise. Then the spectra of both $A$ and $B$ consist of 0 and 1, each of infinite multiplicity. Consequently $B=U^{*} A U$ for a unitary $U$ and moreover $A-B=H=\left(h_{i j}\right)$, where $h_{00}=1$ and $h_{i j}=0$ otherwise. Clearly $\|H\|=1$ and $\delta(A)=1-0=1$, where $\delta(A)$ is defined in Theorem 1 . The particular matrices $A, B$ thus constructed are singular. However, it is clear that they can be replaced by, say, the non-singular positive matrices $A+I$ and $B+I$.

Furthermore, whenever (2) and (3) hold with an operator $A \geqslant 0$ (as, for example, in the preceding paragraph) one can take the unique non-negative self-adjoint square root $P$ of $A$ and form the operator $D=P U$. Then

$$
H=A-B=A-U^{*} A U=D D^{*}-D^{*} D,
$$

so that $D$ is semi-normal. It should be noted however that $D$ need not be non-singular.

Theorem 4. If $H$ of (8) satisfies $H \geqslant 0$ and $H \neq 0$, if $D D^{*}$ differs from a completely continuous operator by a multiple of the identity and if $z=|z| e^{i \theta}$ satisfies $|z|<\|H\| / \delta$, where $\delta=\delta(D(\theta))$ denotes the difference of the maximum and minimum points of the essential spectrum of $D(\theta)=e^{-i \theta} D+e^{i \theta} D^{*}$, then $D_{z} D_{z}{ }^{*}$ and $D_{z}{ }^{*} D_{z}$, where $D_{z}=D-z I$, cannot be unitarily equivalent.

Proof of Theorem 4. First, note that (8) holds if $D$ is replaced by $D_{z}$ so that

$$
H=D_{z} D_{z}^{*}-D_{z}^{*} D_{z} \text {. }
$$

Now if $D_{z} D_{z}{ }^{*}$ and $D_{z}{ }^{*} D_{z}$ are unitarily equivalent, then, by Theorem $3,\|H\| \leqslant \delta\left(D_{z} D_{z}{ }^{*}\right)$. Since

$$
D_{z} D_{z}^{*}=D D^{*}+|z|^{2} I-\bar{z} D-z D^{*}
$$

and since, by hypothesis, $D D^{*}=t I+C$, where $C$ is completely continuous, it follows from Weyl's theorem [7] that the essential spectrum of $D_{z} D_{z}{ }^{*}$ is identical with that of

$$
\left(|z|^{2}+t\right) I-\bar{z} D-z D^{*}
$$

But the essential spectrum of this operator is simply that of $-\bar{z} D-z D^{*}=-|z| D(\theta)$ displaced by the amount $|z|^{2}+t$ and the proof of Theorem 4 is now complete.

A corollary of Theorem 4 is

Theorem 5. If $H$ of (8) satisfies $H \geqslant 0$ and $H \neq 0$, if $D D^{*}$ differs from a completely continuous operator by a multiple of the identity and if $|z| \leqslant \frac{1}{4}\|H\| /\|D\|$, then $z$ is in the spectrum of $D$.

Proof of Theorem 5. Since not only the essential spectrum but even the spectrum of any self-adjoint operator $G$ is contained in an interval of length $2\|G\|$, it follows that 


$$
\delta(D(\theta)) \leqslant 2\|D(\theta)\| \leqslant 4\|D\| .
$$

Hence, if $|z|<\frac{1}{4}\|H\| /\|D\|$, then $D_{z} D_{z}{ }^{*}$ and $D_{z}{ }^{*} D_{z}$ are not unitarily equivalent and so $z$ must surely be in the spectrum of $D$. The sign $\leqslant$ occurring in the theorem, rather than just $<$, follows from the fact that the spectrum is a closed set.

If $V$ is an isometric but not unitary operator, so that $H=V^{*} V-V V^{*} \geqslant 0, H \neq 0$, where $V^{*} V=I$, Theorem 5 implies (with $D=V^{*}$ ) that the disk $|z| \leqslant \frac{1}{4}$ is in the spectrum of $V^{*}$ (hence of $V$ ). Actually it is easy to show that the entire disk $|z| \leqslant 1$ is in the spectrum ; cf. $[4$, p. 1650$]$.

5. Remarks. It will remain undecided whether the hypothesis $|z| \leqslant \frac{1}{4}\|H\| /\|D\|$ in Theorem $5 \mathrm{can}$, as in the isometric non-unitary case, be weakened to $|z| \leqslant\|H\| /\|D\|$. An analogous situation exists for the real part $\frac{1}{2}\left(D+D^{*}\right)$ of a semi-normal operator for which it is known [4] that, if $H \geqslant 0$ in (8),

$$
\|H\| \leqslant 2\|D\| s
$$

where $s$ denotes the measure of the spectrum of $\frac{1}{2}\left(D+D^{*}\right) \equiv J$, and for which it is undecided whether $\|H\| \leqslant \frac{1}{2}\|D\| s$ can also be claimed. (In the isometric operator example mentioned one has $\|H\|=\frac{1}{2}\|D\| s$; cf. [4, p. 1651].)

Actually the inequality $\|H\| \leqslant 4\|D\| s$, rather than (9), was stated in [4] but it is clear from the proof as given in [3] and applied to the case at hand, that the refinement (9) holds. In fact, it follows from (8) that $\frac{1}{2} H=D J-J D$. Hence, if $J=\int \lambda d E(\lambda)$, then, proceeding as in [3], one obtains

$$
\frac{1}{2} \Delta E H \Delta E=\Delta E D \int_{\Delta}\left(\lambda-\lambda_{0}\right) d E-\int_{\Delta}\left(\lambda-\lambda_{0}\right) d E D \Delta E,
$$

where $\Delta$ denotes a real interval and $\lambda_{0}$ is any point of $\Delta$. If $\lambda_{0}$ is chosen to be the mid-point of $\Delta$, the argument of [3] then yields the desired inequality (9). It can be remarked here that the 4 in both Theorem 2 and Corollary 3 of [4] can be replaced by 2 .

\section{REFERENCES}

1. T. Kato, On finite-dimensional perturbations of self-adjoint operators, J. Math. Soc. Japan, 9 (1957), 239-249.

2. T. Kato, Perturbation of continuous spectra by trace class operators, Proc. Japan Academy, 33 (1957), 260-264.

3. C. R. Putnam, On commutators and Jacobi matrices, Proc. American Math. Soc., 7 (1956), 1026-1030.

4. C. R. Putnam, On semi-normal operators, Pacific J. Math. 7 (1957), 1649-1652.

5. C. R. Putnæm, Commutators and absolutely continuous operators, Trans. American Math. Soc., 87 (1958), 513-525.

6. M. Rosenblum, Perturbation of the continuous spectrum and unitary equivalence, Pacific $J$. Math., 7 (1957), 997-1010.

7. H. Weyl, Ùber beschränkte quadratische Formen, deren Differenz vollstetig ist, Rend. Oirc. Math. Palermo, 27 (1909), 373-392.

\section{Purdue University}

LaFayetTe

INDIANA, U.S.A. 